\title{
Forced precession models for six erratic comets
}

\author{
M. Królikowska ${ }^{1}$, G. Sitarski1 ${ }^{1,2}$, and S. Szutowicz ${ }^{1}$ \\ 1 Space Research Centre, Polish Academy of Sciences, Bartycka 18A, 00-716 Warsaw, Poland \\ 2 Institute of Theoretical Physics, University in Białystok, Lipowa 41, 15-424 Białystok, Poland
}

Received 26 June 2000 / Accepted 6 December 2000

\begin{abstract}
The nongravitational motion of six "erratic" short-period comets is studied on the basis of published astrometric observations. We present the precession models which successfully link all the observed apparitions of the comets: 16P/Brooks 2, 21P/Giacobini-Zinner, 31P/Schwassmann-Wachmann 2, 32P/Comas Solá, 37P/Forbes and $43 \mathrm{P} /$ Wolf-Harrington. We used the Sekanina's forced precession model of the rotating cometary nucleus to include the nongravitational terms into equations of the comet's motion. Values of six basic parameters (four connected with the rotating comet nucleus and two describing the precession of spin-axis of the nucleus) have been determined along the orbital elements from positional observations of the comets. The solutions were derived with additional assumptions which introduce instantaneous changes of modulus of reactive force, and of maximum cometary activity with respect to perihelion time. The present precession models impose some constraints on sizes and rotational periods of cometary nuclei. According to our solutions the nucleus of $21 \mathrm{P} /$ Giacobini-Zinner with oblateness along the spin-axis of about 0.29 (equatorial to polar radius of 1.41 ) is the most oblate among six investigated comets.
\end{abstract}

Key words. solar system - comets - individual - 16P/Brooks 2, 21P/Giacobini-Zinner, 31P/SchwassmannWachmann 2, 32P/Comas Solá, 37P/Forbes and 43P/Wolf-Harrington

\section{Introduction}

A vast majority of the known short-period comets are subject to nongravitational forces (Marsden \& Williams 1997). The method to determine nongravitational effects in the comet's orbital motion was proposed by Marsden et al. (1973), who assumed that the three components of a nongravitational force acting on the comet have the form:

$F_{i}=A_{i} g(r) \quad A_{i}=$ const for $i=1,2,3$

where $F_{1}, F_{2}, F_{3}$ represent the radial, transverse and normal component of the nongravitational force, respectively. The analytical function $g(r)$ simulates the ice sublimation rate as a function of the heliocentric distance $r$. At least three consecutive apparitions are needed to determine the "constant" nongravitational parameters $A_{1}, A_{2}, A_{3}$ effective within investigated time interval. To examine whether these parameters are really constant or not, it is necessary to combine sets of three (at least) consecutive apparitions.

In most short-period comets long-term nongravitational effects are constant or slowly changing with time, however, in some comets these effects seem to be strongly variable (see Fig. 2 in Sekanina 1993a). Then, rapidly varying nongravitational perturbations are rather irregular

Send offprint requests to: M. Królikowska, e-mail: mkr@cbk. waw.pl (as in the spectacular case of Comet 21P/GiacobiniZinner), but sometimes may show systematic trends in the motions $(32 \mathrm{P} /$ Comas Sola and $37 \mathrm{P} /$ Forbes cases). It seems that some discontinuities are present in otherwise continuous nongravitational perturbations, thus comets exhibiting such a behaviour are called "erratic" comets (Marsden \& Sekanina 1971). From rich sample of known erratic short-period comets six are investigated in detail in this paper. Four of them, 31P/Schwassmann-Wachmann 2, 32P/Comas Solá, $37 \mathrm{P} /$ Forbes, and $43 \mathrm{P} /$ Wolf-Harrington, have been observed for almost seventy-year long time interval since their discoveries (see Table 1 and Fig. 1); the next two - 16P/Brooks 2 and 21P/Giacobini-Zinner - have been observed at 14 and 13 returns, respectively, for almost a century. All of them have experienced close approaches to Jupiter; in Fig. 2 we present two orbits for each comet to show changes of heliocentric orbits caused by approaches to Jupiter (more details are given in Sect. 5).

Various interpretations have been proposed to explain the long-term variations in nongravitational perturbations of comet's orbital motion. In this paper the nongravitational motion of these erratic comets during their whole observational intervals assuming that sublimation of the rotating and precessing icy cometary nucleus is a source of nongravitational effects and their temporal variations. 

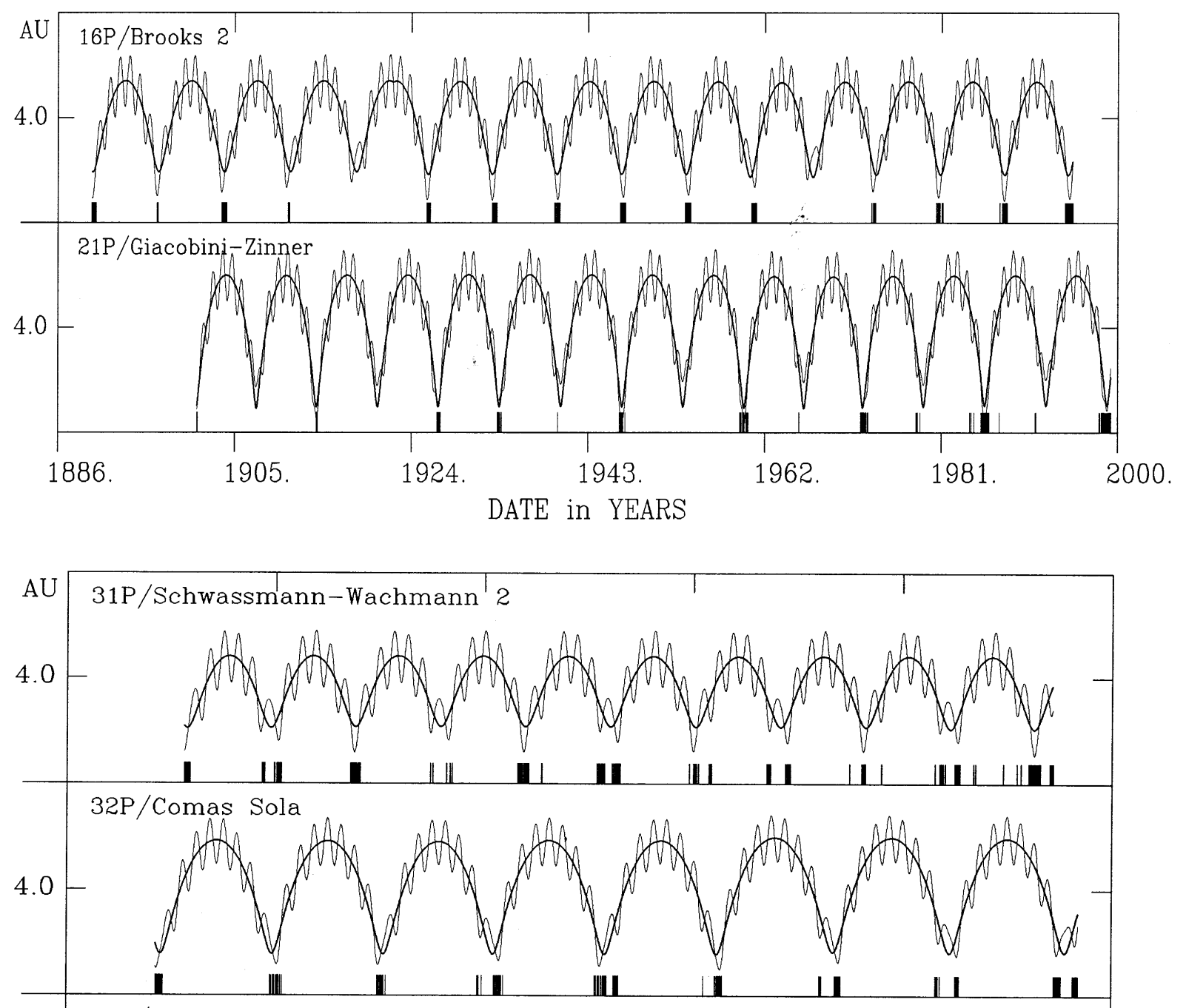

$37 \mathrm{P} /$ Forbes
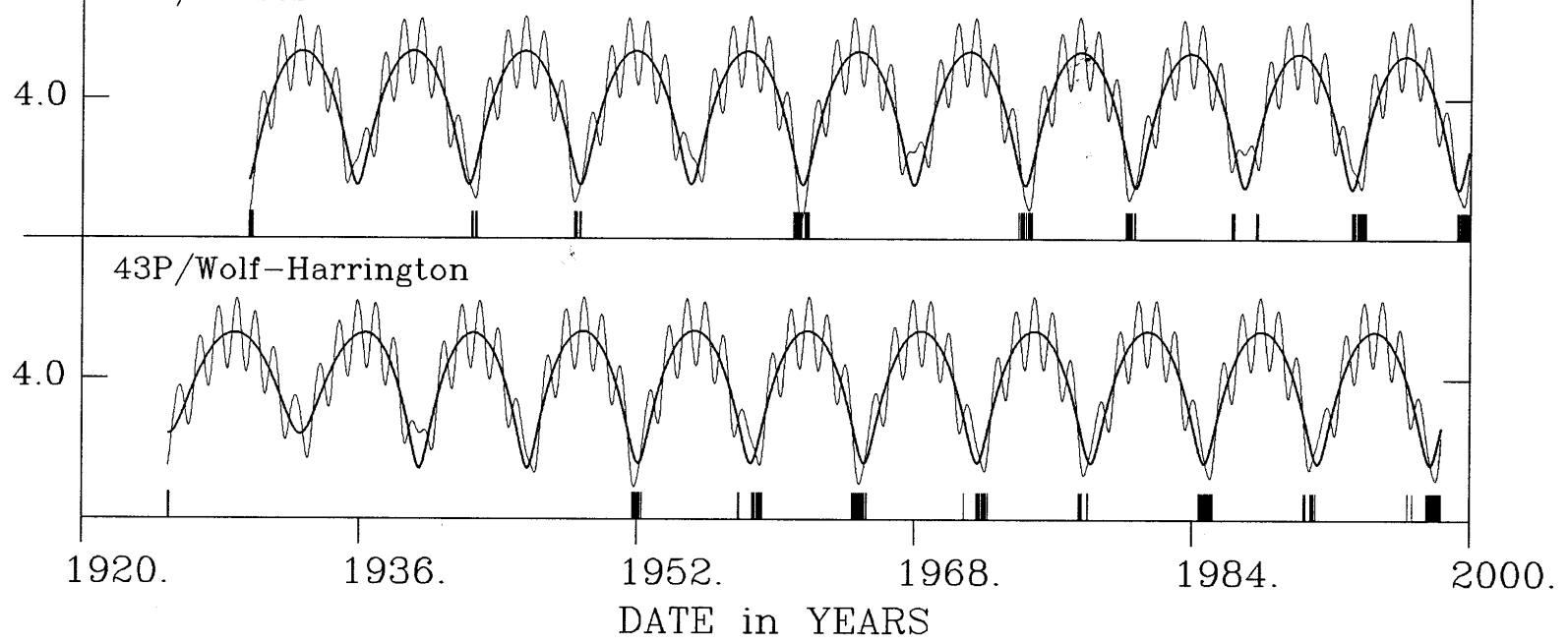

Fig. 1. Temporal variation of the cometary distance from the Sun (thick solid curves) and from the Earth (thin solid curves) for six investigated comets. The distributions of observations with respect to perihelion passages and approaches of the comets to the Earth are shown on the bottom of each graph

We will show that it is possible to link all apparitions of each comet on the basis of forced precession model with physically reasonable parameters. Thus, we conclude that the forced precession model of the rotating nonspherical cometary nucleus adequately explains variations of the nongravitational effects observed in investigated "erratic" 


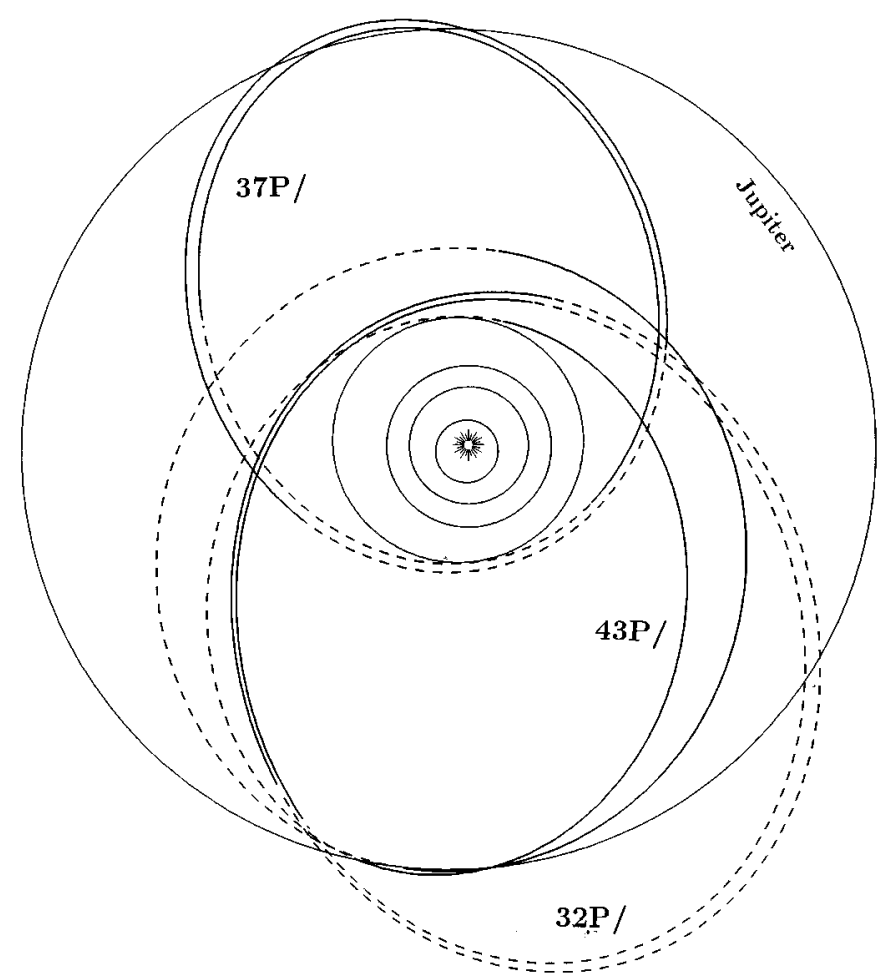

(a)

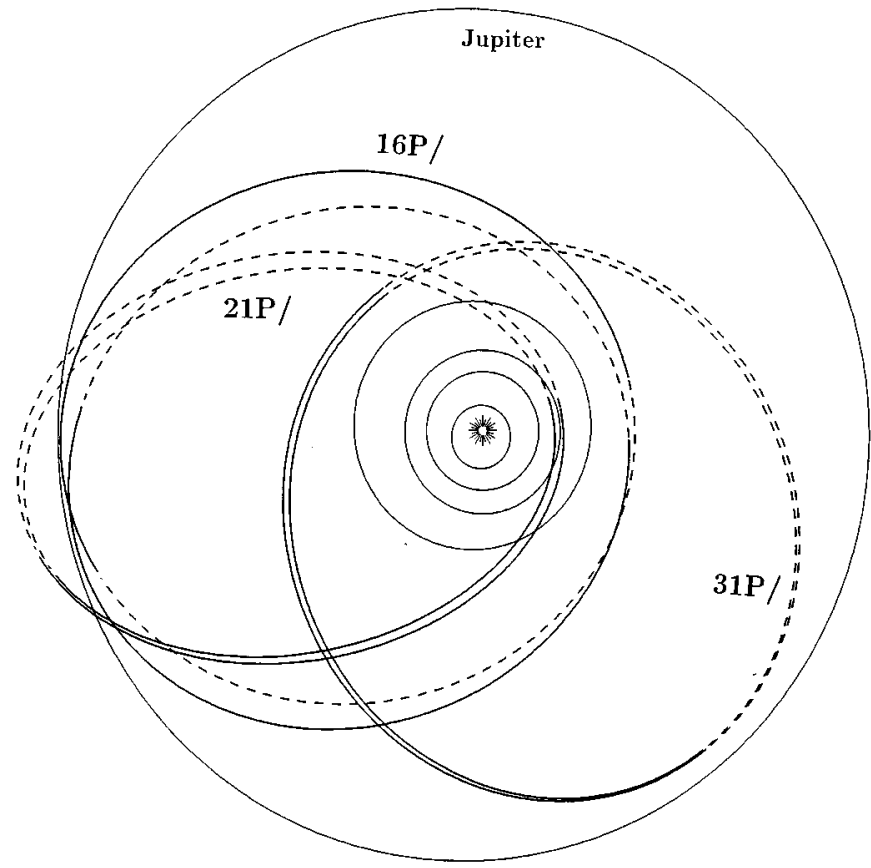

(b)

Fig. 2. Changes of the orbits of six comets caused by approaches to Jupiter; the dashed curves denote these parts of cometary orbit which are placed under the ecliptic plane

comets and is suitable to make predictions of the future returns. Preliminary analysis including five of six comets discussed here, was published elsewhere (Królikowska et al. 1999). These investigations are a continuation of studies of nongravitational effects undertaken by Sitarski and collaborators (e.g. Sitarski 1992, 1994b; Królikowska \& Sitarski 1996; Królikowska et al. 1998a, 1998b; Królikowska \& Szutowicz 1999; Szutowicz 1999, 2000).

\section{Observational material}

We collected all astrometric observations of the periodic comets 31P/Schwassmann-Wachmann 2, 32P/Comas Solá, 37P/Forbes, and 43P/Wolf-Harrington and major part of data for 16P/Brooks 2 and 21P/Giacobini-Zinner. All these comets have been observed at more than seven apparitions. Observations for each apparition were selected according to the objective criteria elaborated by Bielicki \& Sitarski (1991). The number of residuals for some overpopulated apparitions in the observational data were reduced by taking into account normal places (i.e. more than two observations on the same day are replaced by a single average comet position). The mean residuals computed for each apparition separately allowed to calculate the mean residual a priori for the whole observational set of each comet. The mean residual a priori should be compared with the RMS residual resulting from the numerical model of the comet's motion. The global characteristics of observations are presented in Table 2, and distribution of observations with respect to the perihelion passages and approaches of the comets to the Earth are presented graphically in Fig. 1.

\section{Marsdenian nongravitational parameters}

In Table 3 we present the nongravitational parameters $A_{1}, A_{2}, A_{3}$ determined as constant values within sets of three (or four) consecutive apparitions for each of six erratic comets. Temporal variations of most sensitive nongravitational parameter $A_{2}$ are plotted in Fig. 3 in open circles and thin lines. For three investigated comets (21P/Giacobini Zinner, 32P/Comas Solá and 37P/Forbes) the deceleration detected in the motion changed into acceleration; remaining three comets have negative and variable $A_{2}$. In our investigations the estimates of $A_{1}, A_{2}$ and $A_{3}$ can be treated only as a qualitative indication of changes of nongravitational effects within a whole observational time interval, especially at the moments of unexpected changes of trends in behaviour are important for more detailed and more accurate calculations presented in Sects. 4-5. In other words, we show that long-term behaviour of nongravitational parameters resulting from forced precession models may differ from the nongravitational parameters obtained in Marsden's formalism. 
Table 1. Global range of orbital parameters (perihelion distance, $q$, eccentricity, $e$, and inclination, $i$ ) and close encounters with Jupiter for six erratic comets

Range of orbital parameters

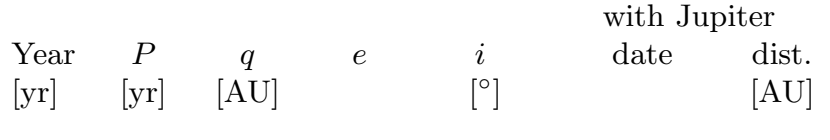

\begin{tabular}{lllllll}
\hline \multicolumn{7}{c}{$\mathbf{1 6 P} /$ Brooks 2 } \\
1886 & 37.5 & 5.453 & 0.513 & 7.134 & July 1886 & .001 \\
1911 & 7.10 & 1.963 & 0.469 & 6.070 & Feb. 1922 & 0.12 \\
1994 & 6.89 & 1.843 & 0.491 & 5.541 & & \\
\hline \multicolumn{7}{c}{$\mathbf{2 1 P} /$ Giacobini-Zinner } \\
1887 & 7.00 & 1.241 & 0.661 & 33.408 & Oct. 1898 & 0.19 \\
1900 & 6.46 & 0.932 & 0.732 & 29.830 & Sep. 1969 & 0.57 \\
1972 & 6.52 & 0.994 & 0.715 & 31.703 & & \\
\hline
\end{tabular}

\begin{tabular}{lllllll}
\multicolumn{7}{c}{ 31P/Schwassmann-Wachmann 2 } \\
1920 & 9.29 & 3.565 & 0.194 & 0.741 & & \\
1994 & 6.39 & 2.070 & 0.399 & 3.753 & Mar. 1926 & 0.18 \\
2002 & 8.72 & 3.408 & 0.195 & 4.550 & Mar. 1997 & 0.25 \\
\hline
\end{tabular}

\begin{tabular}{llllllll}
\multicolumn{8}{c}{ 32P/Comas Solá } \\
1910 & 9.35 & 2.152 & 0.515 & 18.707 & May 1912 & 0.18 \\
1927 & 8.52 & 1.772 & 0.575 & 13.766 \\
1996 & 8.83 & 1.846 & 0.568 & 12.917 & & &
\end{tabular}

\begin{tabular}{lllllll}
\multicolumn{7}{c}{ 37P/Forbes } \\
1929 & 6.38 & 1.528 & 0.556 & 4.645 & & \\
1987 & 6.26 & 1.475 & 0.566 & 4.671 & Aug. 1990 & 0.34 \\
1993 & 6.13 & 1.447 & 0.568 & 7.159 & & \\
\hline
\end{tabular}

\begin{tabular}{lllllll}
\multicolumn{7}{c}{ 43P/Wolf-Harrington } \\
1925 & 7.60 & 2.428 & 0.372 & 23.684 & & \\
1939 & 6.20 & 1.448 & 0.571 & 18.322 & June 1936 & 0.12 \\
1952 & 6.50 & 1.599 & 0.541 & 18.493 & Jan. 1948 & 0.72 \\
1997 & 6.46 & 1.581 & 0.544 & 18.510 & &
\end{tabular}

\section{Forced precession model}

We used the Sekanina's $(1984,1988)$ forced precession model of a rotating cometary nucleus to include the nongravitational terms into equations of comet's motion. Values of six basic parameters (four connected with the rotating comet nucleus and two describing the precession of spin-axis of the nucleus) have been determined along with the orbital elements from positional observations of the comets. Those six parameters are:

- modulus of the reactive force divided by $g(r)$, A, where $A=\left(A_{1}^{2}+A_{2}^{2}+A_{3}^{2}\right)^{1 / 2}$

- lag angle $\eta$ of the maximum outgassing behind subsolar meridian;
Table 2. Global characteristics of observations of six erratic comets. For Comet 16P/Brooks 2 observations till 1939 apparition were simulated; also the observations for 1926 and 1939/40 apparitions in the Comet 21P/Giacobini-Zinner case, were simulated; thus the mean residuals a priori for both of them are not given

$\begin{array}{llcc}\text { Comet No of } & \text { No } & \text { No } & \text { Mean } \\ \text { design. apparitions } & \text { Observation interval } & \begin{array}{c}\text { of } \\ \text { of }\end{array} & \text { res. } \\ & & \text { obs. } & \text { res. }\end{array}$

\begin{tabular}{|c|c|c|c|c|}
\hline $16 \mathrm{P}$ & 14 & 1889 Aug. $29-1995$ Feb. 24595 & 961 & $1^{\prime \prime} 78$ \\
\hline $21 \mathrm{P}$ & 13 & 1900 Dec. $24-1999$ Apr. 301589 & 2529 & $2 . \prime 04$ \\
\hline $31 \mathrm{P}$ & 11 & 1929 Jan. $4-1995$ July 4485 & 921 & $1^{\prime \prime} 31$ \\
\hline $32 \mathrm{P}$ & 9 & 1926 Nov. $\quad 4-1997$ June 25582 & 939 & 1." 41 \\
\hline $37 \mathrm{P}$ & 9 & 1929 Aug. $22-1999$ Nov. 11286 & 553 & $1^{\prime \prime} 15$ \\
\hline $43 \mathrm{P}$ & 9 & 1924 Dec. $22-1998$ May 26322 & 628 & $1^{\prime \prime} 23$ \\
\hline
\end{tabular}

- obliquity $I$ of the orbit plane to the cometary equator;

- cometocentric longitude $\phi$ of the Sun at perihelion;

- nucleus oblateness $s=1-R_{\mathrm{b}} / R_{\mathrm{a}}$, where $R_{\mathrm{a}}$ and $R_{\mathrm{b}}$ are equatorial and polar radii, respectively;

- precession factor $f_{\mathrm{p}}$ which depends on a rotation period and a nucleus size.

For the rotating cometary nucleus the orbital components of nongravitational force, $F_{i}$ are functions of time. Thus, $F_{i}$ are related to the angular parameters $\eta, I, \phi$ of the rotating nucleus by:

$$
F_{i}(t)=A \cdot C_{i}(\eta, I(t), v(t)+\phi(t)) \cdot g(r) \text { for } i=1,2,3
$$

where $v$ is the true anomaly of the comet and $C_{i}$ denote the direction cosines, which are time-dependent due to the orbital and precessional motion of the comet. Expressions for time variations of the angles $I$ and $\phi$, the precession rate, and the direction cosines, $C_{i}$, of the nongravitational force are given by Królikowska et al. (1998b), (hereafter referred as KSS) together with a detailed description of the method of computations. We adopt the Marsden's water production curve, $g(r)$, whose analytical form is useful for orbital computations.

To find satisfactory solutions we have to accept the following additional assumptions characterizing a specific physical behaviour of these comets:

- 1. Maximum of cometary activity with respect to perihelion can be asymmetric;

- 2a. The time shift parameter which describes displacement of maximum activity with respect to the perihelion could change its value during investigated apparitions; and/or

- $\mathbf{2 b}$. Real activity of the comet change during the considered time interval.

The first assumption introduces an additional unknown parameter $\tau$ representing a time shift of the maximum of 
Table 3. Nongravitational parameters $A_{1}, A_{2}, A_{3}$ determined by linking three or four consecutive apparitions. The mean residual RMS and number of observations used to improve the orbits are given in the last two columns

\begin{tabular}{|c|c|c|c|c|c|}
\hline Appearances & $A_{1}$ & \multicolumn{2}{|c|}{$\begin{array}{l}A_{2} \\
10^{-8} \mathrm{AU} / \mathrm{day}^{2}\end{array}$} & $\begin{array}{l}\text { Mean } \\
\text { res }\end{array}$ & $\begin{array}{l}\text { No } \\
\text { of } \\
\text { obs. }\end{array}$ \\
\hline \multicolumn{6}{|c|}{$16 P /$ Brooks 2} \\
\hline $1889 / 96 / 03$ & $4.11 \pm .92$ & $-.3243 \pm .0021$ & $-.271 \pm .229$ & $2{ }^{\prime \prime} 81$ & 114 \\
\hline $1896 / 03 / 11$ & $2.26 \pm .41$ & $-.2870 \pm .0019$ & $-.386 \pm .097$ & $1 !^{\prime \prime} 47$ & 108 \\
\hline $1903 / 11 / 25$ & $0.978 \pm .154$ & $-.2410 \pm .0030$ & $+.564 \pm .130$ & $1 .^{\prime \prime} 44$ & 118 \\
\hline $1911 / 25 / 32$ & $1.01 \pm .15$ & $-.2190 \pm .0066$ & $+.523 \pm .103$ & $1 !^{\prime \prime} 41$ & 110 \\
\hline $1932 / 39 / 46$ & $0.357 \pm .220$ & $-.2517+.0012$ & $+.200 \pm .093$ & $1^{\prime \prime} 89$ & 81 \\
\hline $1939 / 46 / 53$ & $1.34 \pm .27$ & $-.2341 \pm .0011$ & $+.121 \pm .080$ & $1^{\prime \prime} 76$ & 71 \\
\hline & $1.25 \pm .17$ & $-.2055 \pm .0077$ & $+.081 \pm .062$ & $1{ }^{\prime \prime} 73$ & 63 \\
\hline & $1.35+.14$ & $-.2051+.0020$ & $+.169+.061$ & $1^{\prime \prime} 43$ & 54 \\
\hline $1960 / 74 / 80$ & $0.685 \pm .229$ & $-.1614 \pm .0020$ & $+.177 \pm .076$ & $1{ }^{\prime \prime} 67$ & 40 \\
\hline $1974 / 80 / 87$ & $0.584 \pm .235$ & $-.1354 \pm .0019$ & $+.253 \pm .073$ & $2 . \prime 03$ & 90 \\
\hline $1980 / 87 / 94$ & $0.234 \pm .163$ & $-.1574 \pm .0005$ & $+.112 \pm .047$ & 1.' 61 & 181 \\
\hline \multicolumn{6}{|c|}{ 21P/Giacobini-Zinner } \\
\hline $1900 / 13 / 26$ & $0.632 \pm .136$ & $\begin{array}{r}+.0348 \pm .0001 \\
\end{array}$ & $-.037 \pm .095$ & $4 .^{\prime \prime} 07$ & 205 \\
\hline 1913 & $403 \pm .072$ & $+.0351 \pm .0003$ & $+.107 \pm .080$ & $3 .^{\prime \prime} 85$ & 197 \\
\hline 1933 & $0.179 \pm .055$ & $\begin{array}{r}+.0376 \pm .0023 \\
\end{array}$ & $-.069 \pm .055$ & $2 . \prime 08$ & 63 \\
\hline & $278 \pm .016$ & & $-.017 \pm .023$ & $1 .^{\prime \prime} 71$ & 85 \\
\hline 1946 & $244 \pm .011$ & $+.1063 \pm .0001$ & $-.169 \pm .015$ & $2 .^{\prime \prime} 44$ & 180 \\
\hline & $0.357 \pm .021$ & $-.0182 \pm .0005$ & $+.132 \pm .021$ & $2 . \prime 25$ & 144 \\
\hline & $8 \pm .015$ & & $-.077 \pm .016$ & $1 !^{\prime \prime} 81$ & 314 \\
\hline & $146 \pm .038$ & -.06 & $-.148 \pm .019$ & $1 \stackrel{\prime \prime}{\prime \prime} 30$ & 228 \\
\hline 1985/92/99 & $0.218 \pm .010$ & $-.0512 \pm .0018$ & $-.022 \pm .012$ & $1^{\prime \prime} 84$ & 419 \\
\hline \multicolumn{6}{|c|}{ 31P/Schwassmann-Wachmann 2} \\
\hline $29 / 35 / 42 / 48$ & $0.863 \pm .225$ & $-.1984 \pm .0033$ & $-.082 \pm .074$ & $1^{\prime \prime} 34$ & 83 \\
\hline & \pm .05 & & $2 \pm$. & $1 .{ }^{\prime \prime} 12$ & 103 \\
\hline & $0.805 \pm .082$ & & $+.076 \pm .046$ & $1 !^{\prime \prime} 11$ & 121 \\
\hline $48 / 55 / 61 / 68$ & $1.347 \pm .052$ & $-.1672 \pm .0014$ & $\begin{array}{r}.007 \pm .053 \\
+.007\end{array}$ & $1 .^{\prime \prime} 38$ & 116 \\
\hline & $11 \pm .063$ & -.2 r & $-.088 \pm .050$ & $1 .{ }^{\prime \prime} 70$ & 146 \\
\hline & $03 \pm .068$ & & $-.026 \pm .062$ & $2 .^{\prime \prime} 11$ & 125 \\
\hline$/ 81 / 87$ & $1.576 \pm .092$ & $3 \pm .0025$ & $+.040 \pm .088$ & 3 .' 48 & 136 \\
\hline $74 / 81 / 87 / 94$ & $1.306 \pm .031$ & $-.2960 \pm .0009$ & $+.005 \pm .029$ & 1 1! 66 & 273 \\
\hline \multicolumn{6}{|c|}{ 32P/Comas Solá } \\
\hline & $823 \pm .088$ & & $-.054 \pm .045$ & $2 . \prime 02$ & 172 \\
\hline & .055 & & $-.143 \pm .8$ & $1 . \prime 48$ & 125 \\
\hline & $0.744 \pm .051$ & $9 \pm .00$ & $-.130 \pm .027$ & $1 .^{\prime \prime} 33$ & 116 \\
\hline & $691 \pm .046$ & -.0 & $-.211 \pm .0$ & $1{ }^{\prime \prime} 33$ & 126 \\
\hline & .04 & 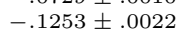 & $2 \pm .030$ & $1 . \prime 52$ & 14 \\
\hline & $05 \pm .104$ & $8 \pm .0010$ & $+.037 \pm .049$ & $1 .^{\prime \prime} 49$ & 114 \\
\hline $1977 / 87 / 96$ & $0.872 \pm .072$ & $-.1125 \pm .0014$ & $-.090 \pm .054$ & $1 . \prime 41$ & 162 \\
\hline \multicolumn{6}{|c|}{$37 P /$ Forbes } \\
\hline & $0.416 \pm .186$ & $+.0805 \pm .00$ & $+.044 \pm .040$ & $1{ }^{\prime \prime} 82$ & 48 \\
\hline & 3 &.+ & \pm .025 & $1 . \prime 56$ & 46 \\
\hline & $0.694 \pm .036$ & -.0 & $-.072 \pm .018$ & $1 .^{\prime \prime} 71$ & 65 \\
\hline & $0.565 \pm .049$ & & $-.036 \pm .022$ & $1{ }^{\prime \prime} 69$ & 68 \\
\hline & $.829 \pm .060$ & -.06 & $-.046 \pm .022$ & $1^{\prime \prime} 26$ & 66 \\
\hline & $0.359 \pm .030$ & $-.0391 \pm .0012$ & $-.023 \pm .018$ & $1 !^{\prime \prime} 14$ & 111 \\
\hline $1987 / 93 / 99$ & $0.409 \pm .027$ & $-.0403 \pm .0013$ & $-.012 \pm .018$ & $1 .^{\prime \prime} 06$ & 167 \\
\hline \multicolumn{6}{|c|}{$43 P /$ Wolf-Harrington } \\
\hline & $0.109 \pm .077$ & & $-.022 \pm .041$ & $1^{\prime \prime} 48$ & 79 \\
\hline & $0.199 \pm .038$ & $-.0513 \pm .001$ & $-.049 \pm .025$ & $1 .{ }^{\prime \prime} 18$ & 91 \\
\hline & $0.343 \pm .034$ & $-.0486 \pm .00$ & $-.031 \pm .023$ & $1{ }^{\prime \prime} 27$ & 77 \\
\hline & $0.370 \pm .084$ & -.04 & $-.013 \pm .039$ & $1{ }^{\prime \prime} 60$ & 59 \\
\hline $1971 / 78 / 84$ & $0.333 \pm .056$ & $-.0096 \pm .0028$ & $-.000 \pm .037$ & $1 .^{\prime \prime} 87$ & 69 \\
\hline & $0.285 \pm .116$ & $-.0178 \pm .0025$ & $-.033 \pm .057$ & $1{ }^{\prime \prime} 86$ & 71 \\
\hline & $0.406 \pm .021$ & $-.0366 \pm .0008$ & $-.009 \pm .015$ & $1 . \prime 12$ & 184 \\
\hline
\end{tabular}

$g(r)$ with respect to the perihelion time. Thus, the function $g(r)$ is then replaced by $g\left(r^{\prime}\right)$, where $r^{\prime}=r(t-\tau)$ (Sekanina 1988; Sitarski 1994a). The time shift $\tau$ can be determined together with the basic parameters from the forced precession model, but only for Comet 37P/Forbes case the model based on seven unknown parameters (basic six parameters plus $\tau$ ) proved to be reliable. For the remaining comets it was necessary to introduce the assumptions $2 \mathrm{a}$ and/or $2 \mathrm{~b}$. The postulated changes can be modelled by setting the discontinuities of parameters $A$

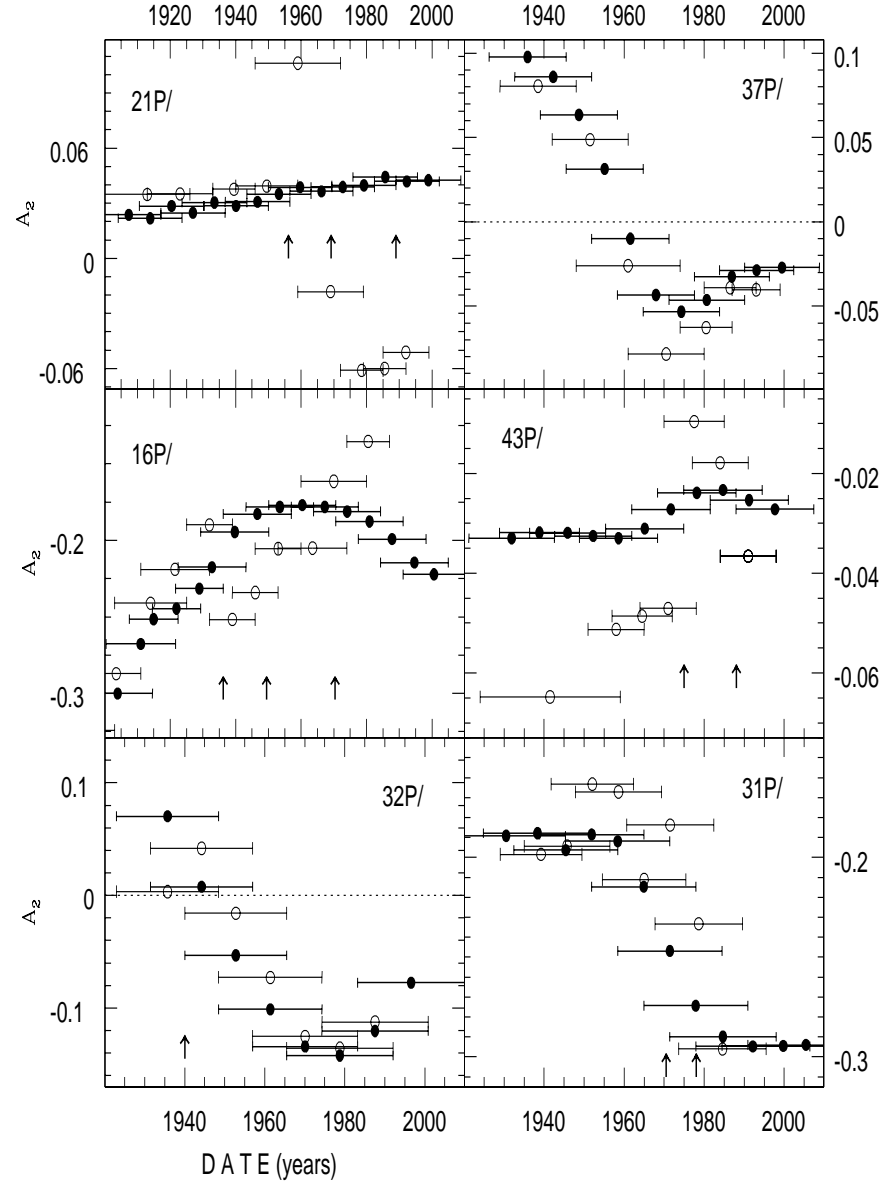

Fig. 3. Temporal variations in the nongravitational parameter $A_{2}$ for six short-period comets. The open circles represent values of $A_{2}$ determined as constant values within sets of at least three consecutive apparitions (see Table 3); these circles are referred to the middle of the time intervals covered by calculations (which are shown as thin solid horizontal lines). The solid circles are the mean (e.g. $\bar{A}_{2}$ from Eq. (3)) averaged over three consecutive revolutions around the Sun) values of $A_{2}$ (e.g. resulting from ours forced precession models discussed in the Sects. 3-5). Solid circles are drawn on the middle of thick dashed horizontal lines which represent the time interval taking into account for $A_{2}$ averaging. Upright arrows indicate the moments of discontinuities of $\tau$ (and also $A$ in $21 \mathrm{P} /$ case) introduced to the forced precession models (see Sect. 4 and Table 4 )

and/or $\tau$ close to selected moments of aphelion passages $\left(t_{b 1}, t_{b 2}\right)$ :

$$
A=\left\{\begin{array}{l}
A^{(1)} \text { for } t<t_{b 1} \\
A^{(2)} \text { for } t \geq t_{b 1}
\end{array} \quad \text { and } / \text { or } \tau=\left\{\begin{array}{l}
\tau_{1} \text { for } t<t_{b 2} \\
\tau_{2} \text { for } t \geq t_{b 2}
\end{array} .\right.\right.
$$

Finally, the parameters $A^{(i)}(i=1,2, \ldots), \eta, I_{0}{ }^{1}, \phi_{0}, f_{\mathrm{p}}$, $s$ and $\tau_{k}(k=1,2, \ldots)$ were determined simultaneously with six orbital elements from the observational equations by an iterative least squares process; the number of parameters $A^{(i)}$ and $\tau_{k}$ depend on assumed number of discontinuities of $A$ and $\tau$, respectively.

\footnotetext{
1 Subscript "0" denotes the starting epoch of integration.
} 
Table 4. Orbital elements and physical parameters of the nucleus for forced precession models linking all apparitions of each six comets. Angular elements $\omega, \Omega, i$ are referred to Equinox J2000.0. Parameters $A, A_{1}$ and $A_{2}$ are in units of $10^{-8} \mathrm{AU}^{\mathrm{d}} \mathrm{day}{ }^{2}$, the precession factor $f_{\mathrm{p}}$ is in units of $10^{7}$ day/AU, time shifts $\tau, \tau_{1}, \tau_{2}$ and $\tau_{3}$ are in days, and $t_{b 1}, t_{b 2}, t_{b 3}$ are moments of discontinuities of $A$ or $\tau$

\begin{tabular}{|c|c|c|c|c|c|c|}
\hline & \multicolumn{4}{|c|}{ (a) Prolate spheroid models } & \multicolumn{2}{|c|}{ (b) Oblate spheroid models } \\
\hline & $\begin{array}{c}\text { Wolf-- } \\
\text { Harrington }\end{array}$ & Forbes & Brooks 2 & Comas Solá & $\begin{array}{l}\text { Schwassmann- } \\
\text { Wachmann } 2\end{array}$ & $\begin{array}{l}\text { Giacobini- } \\
\text { Zinner }\end{array}$ \\
\hline Epoch: & 19970820 & 19991208 & 19950224 & 19261101 & 19950704 & 19560217 \\
\hline$T$ & 970929.21850 & 990504.24713 & 940901.04396 & 270322.19729 & 940124.98426 & 530416.36686 \\
\hline$q$ & 1.58182646 & 1.44616915 & 1.84322847 & 1.77244905 & 2.07187392 & 0.98755442 \\
\hline$e$ & 0.54394750 & 0.56841121 & 0.49075850 & 0.57495559 & 0.39993050 & 0.71789222 \\
\hline$\omega$ & $187^{\circ} .13349$ & $310^{\circ} 74872$ & $197^{\circ} .98531$ & 38.50697 & $358^{\circ} 66420$ & 171.82326 \\
\hline$\Omega$ & 254.75651 & 334.35354 & 176.93159 & 66.60484 & 126.07531 & 196.91409 \\
\hline$i$ & 18.51059 & $7^{\circ} .16047$ & 5.54035 & 13.76535 & 3.75704 & 30.84181 \\
\hline$A$ & $+.3634 \pm .0061$ & $+.5584 \pm .0062$ & $+1.1846 \pm .0055$ & $+.7995 \pm 0.0105$ & $+1.399 \pm .011$ & - \\
\hline$A^{(1)}$ & - & - & - & - & - & $+.3031 \pm .0019$ \\
\hline$t_{b 1}$ & - & - & - & - & - & 19560217 \\
\hline$A^{(2)}$ & - & - & - & - & - & $+.3944 \pm .0005$ \\
\hline$\eta$ & $6.36 \pm 0^{\circ} .41$ & $11.89 \pm 0.11$ & $21.35 \pm 0.47$ & $21^{\circ} .70 \pm 0.76$ & $13^{\circ} .18 \pm 0^{\circ} .24$ & $6.53 \pm 0.02$ \\
\hline$I_{0}$ & $125^{\circ} 05 \pm 4.40$ & $87^{\circ} .82 \pm 0.65$ & $118^{\circ} .22 \pm 0.68$ & $50^{\circ} .21 \pm 1.64$ & $160^{\circ} 64 \pm 0.50$ & $25^{\circ} .91 \pm 0.56$ \\
\hline$\phi_{0}$ & $143.47 \pm 14.80$ & $15.78 \pm 4.07$ & $319.49 \pm 0.71$ & $85^{\circ} 06 \pm 3.14$ & $9.16 \pm 4^{\circ} .31$ & $147^{\circ} .24 \pm 0.28$ \\
\hline$f_{\mathrm{p}}$ & $-.3136 \pm .0670$ & $-.4623 \pm .0179$ & $-.08191 \pm 0.00041$ & $-.1735 \pm .0046$ & $+.2865 \pm .0093$ & $+.3460 \pm .0007$ \\
\hline$s$ & $-.0451 \pm .0200$ & $-.0589 \pm .0041$ & $-.0572 \pm 0.0055$ & $-.2886 \pm .0348$ & $+.0923 \pm .0204$ & $+.2926 \pm .0055$ \\
\hline$\tau$ & - & $-8.3254 \pm 0.2563$ & - & - & - & - \\
\hline$\tau_{1}$ & $-17.78 \pm 1.75$ & - & +4.7 & $-53.92 \pm 3.79$ & $+3.291 \pm 1.129$ & $+4.469 \pm .129$ \\
\hline$t_{b 2}$ & 19750101 & - & 19360313 & 19400101 & 19700701 & 19560217 \\
\hline$\tau_{2}$ & $8.394 \pm 0.823$ & - & -20.2 & $+26.25 \pm 1.95$ & $+61.81 \pm 1.08$ & +38.75 \\
\hline$t_{b 3}$ & 19880106 & - & 19490613 & - & 19780101 & 19690301 \\
\hline$\tau_{3}$ & $-11.69 \pm 0.88$ & - & +1.1 & - & $+1.193 \pm 0.756$ & -53.06 \\
\hline$t_{b 4}$ & - & - & 19700514 & - & - & 19881225 \\
\hline$\tau_{4}$ & $\overline{-}$ & $\overline{-}$ & +20.4 & $\overline{ }$ & $\overline{-}$ & -49.38 \\
\hline RMS & 1.! 39 & 1." 47 & $3 . \prime 07$ & $2 . .01$ & 1.. 67 & 4.. 17 \\
\hline
\end{tabular}

In some cases we were able to find the best value of $\tau$ but not as a basic parameter of the mean squares procedure (these $\tau$ 's are given without their mean errors in Table 4)

To show how values of the nongravitational parameters are derived, especially the values of the time shift parameter $\tau$, we take as an example the Comet 16P/Brooks 2 . The procedure is as follows:

(i) Linking observations of the first four apparitions of the comet in $1889 / 90,1896,1903 / 04$, and 1910 , we find the Marsden parameters $A_{1}, A_{2}, A_{3}$ and estimate the preliminary values of $A, \eta, I_{0}, \phi_{0}$;

(ii) Joining subsequently observations of the next apparitions in $1925 / 26,1932 / 33$, and $1939 / 40$ we are also able to determine consecutive values of the parameters $f_{\mathrm{p}}, s$, and $\tau_{1}$. Thus we linked all observations from the interval 1889-1940 characterized by the acceptable mean RMS residual equal to 2 .' 7 , and determine the values of six orbital elements and of seven nongravitational parameters $A, \eta, I_{0}, \phi_{0}, f_{\mathrm{p}}, s, \tau_{1}$. However, basing on that preliminary model of the comet's motion a prediction for the next apparition failed since the predicted RMS residual for the 1946 observations amounted to 76. ., thus it was impossible to obtain a reasonable solution for all the 18891946 observations;

(iii) To add observations of 1946 to a set of 1889-1940 for the orbit improvement we have to find (by the method of "trials and errors" an appropriate value of $\tau_{2}$ and put it after the aphelion time in 1936. Thus we were able to link successfully all observations from 1889-1954 by means of eight nongravitational parameters: $A, \eta, I_{0}, \phi_{0}, f_{\mathrm{p}}, s, \tau_{1}, \tau_{2}$. (iv) To join observations from further apparitions of the comet, we have to add to a set of nongravitational parameters new values of $\tau_{3}$ and $\tau_{4}$ after 1949 and 1970, respectively, like in case of $\tau_{2}$.

\section{Results and limits for the nuclei sizes and rotational periods}

The nongravitational equations of the comet motion have been integrated numerically by the recurrent power series method, taking into account all the planetary perturbations (Sitarski 1979, 1984). Satisfactory solution for each of the six comets were found.

Numerical values of the nongravitational parameters as well as values of the osculating orbital elements are given in Table 4. Solutions with the positive values 


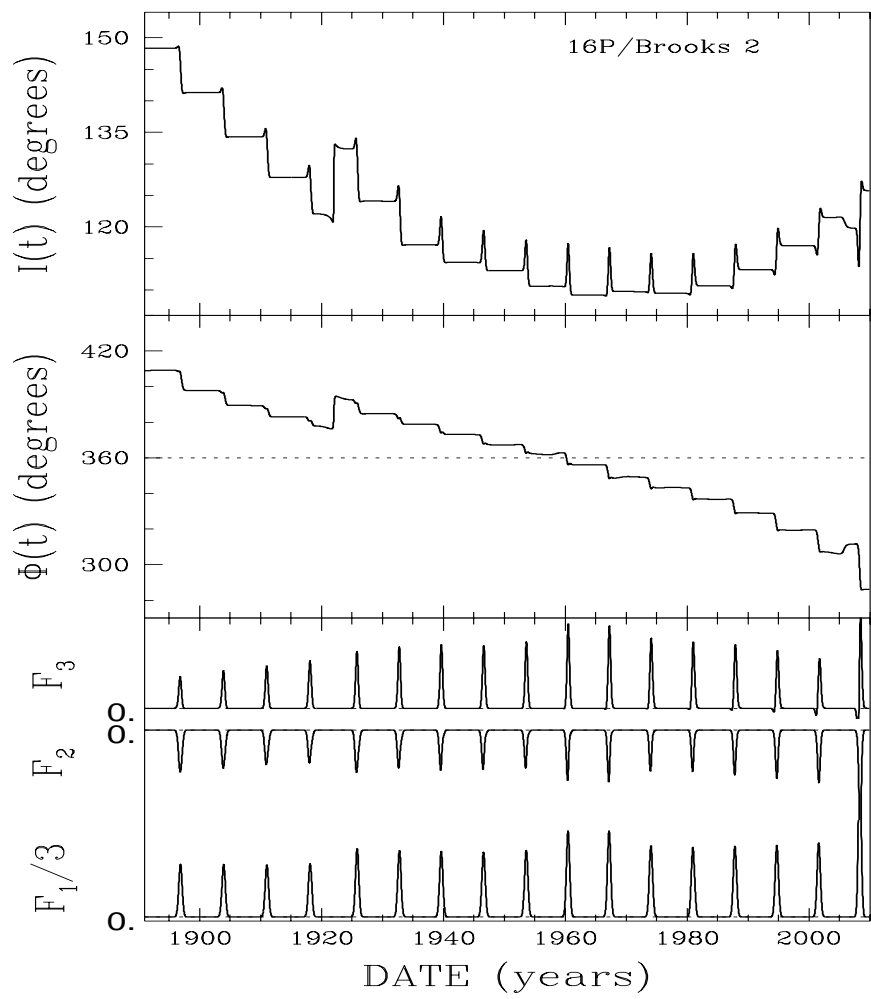

(a)

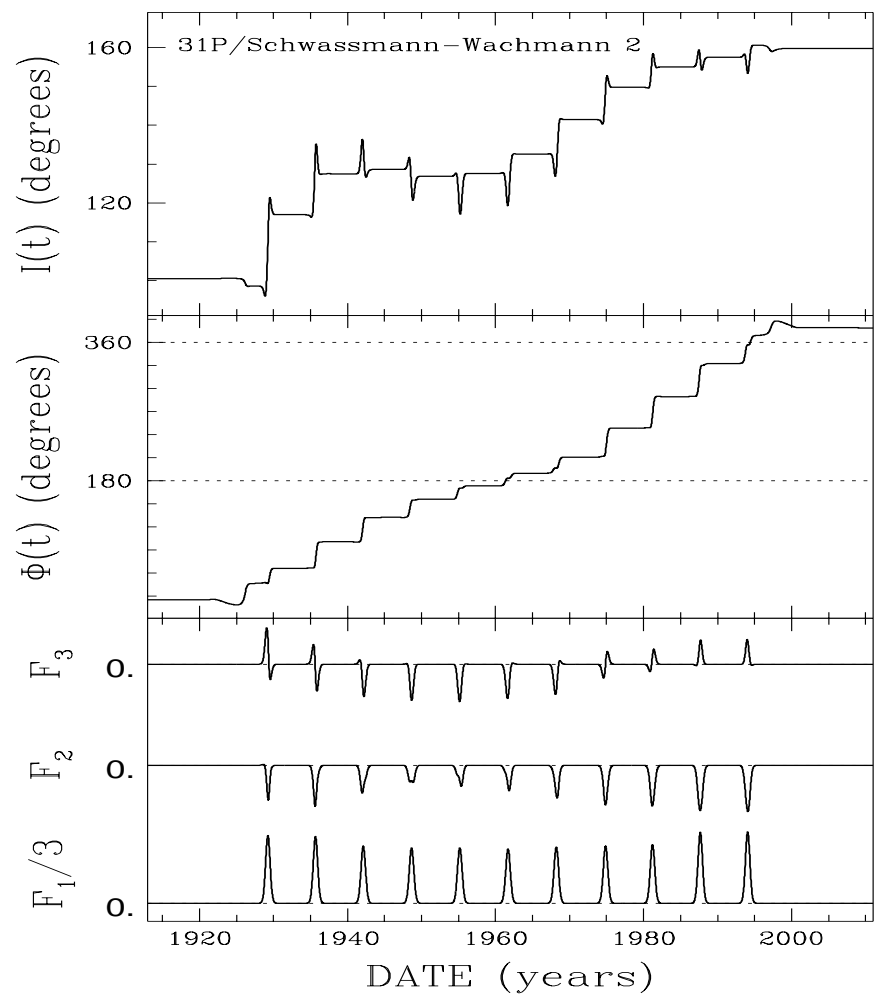

(c)

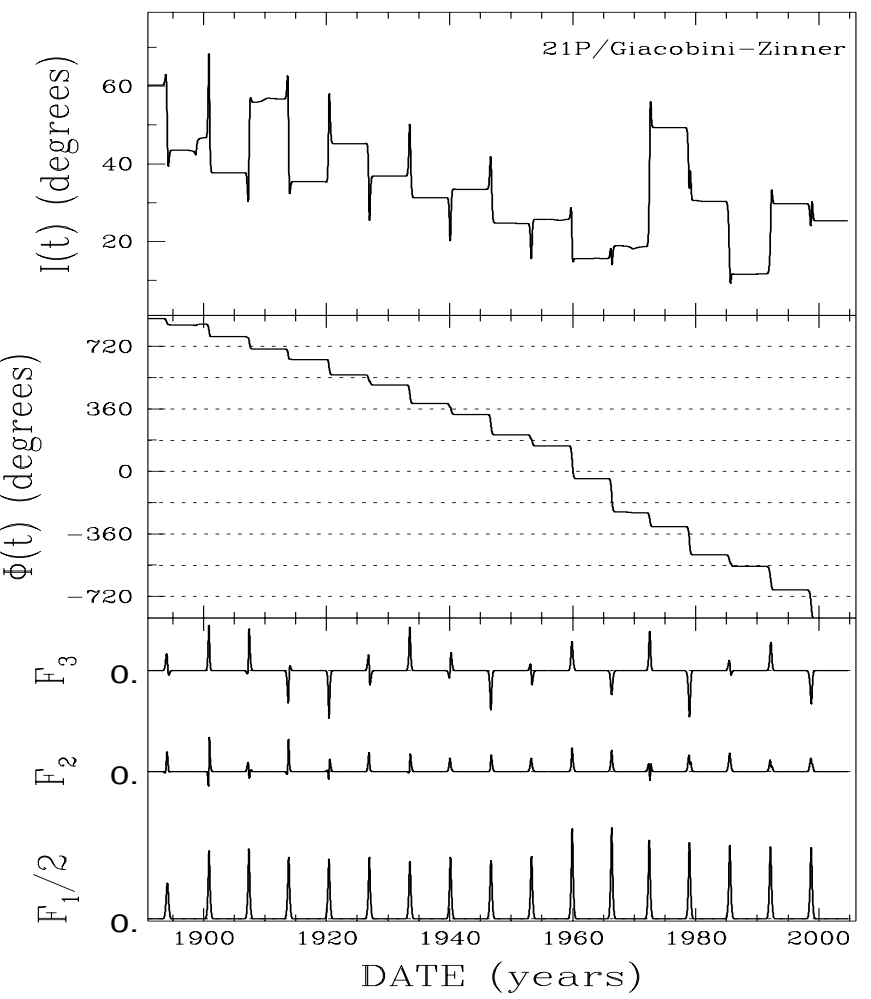

(b)

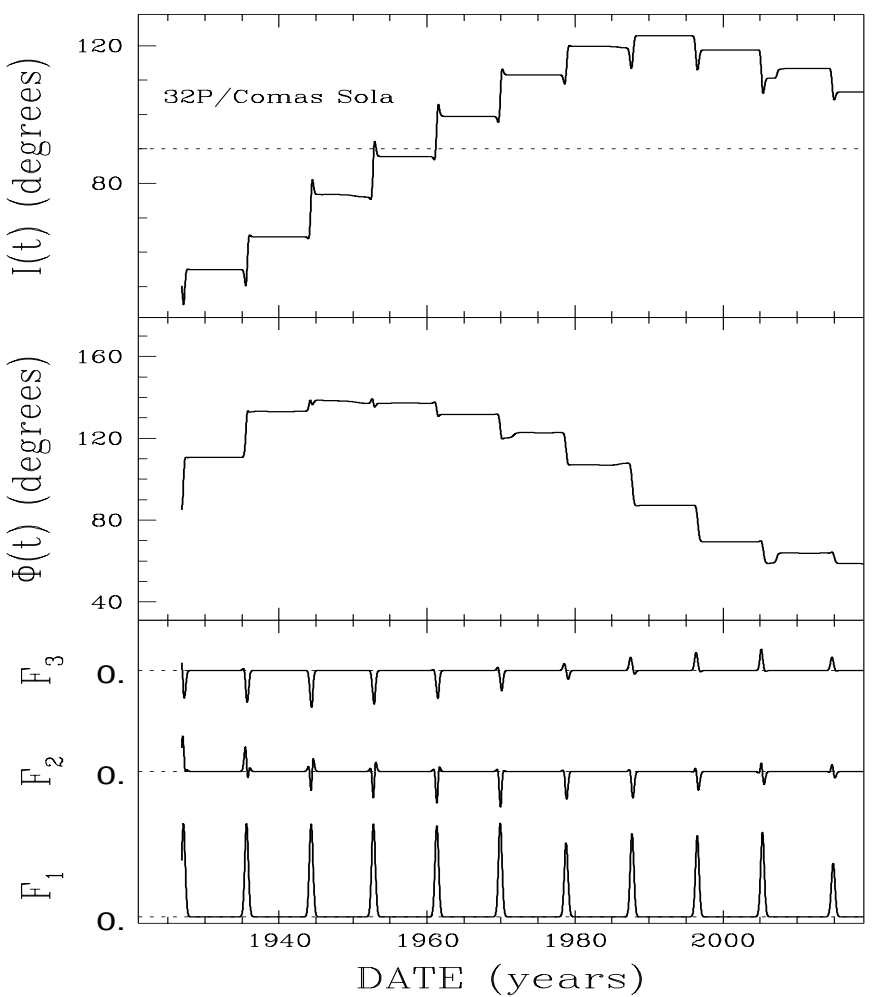

(d)

Fig. 4. Temporal variation of angle $I, \phi$ and components $F_{1}, F_{2}, F_{3}$ of the nongravitational force due to the spin axis precession of cometary nucleus; dashed horizontal line on the upper panels divide models with prograde rotation (consistent with the sense of the cometary orbit around the Sun) when $I<90^{\circ}$ from models with retrograde rotation when $I>90^{\circ}$; dashed horizontal line on the middle panels indicate changes of configuration of poles in the perihelia 


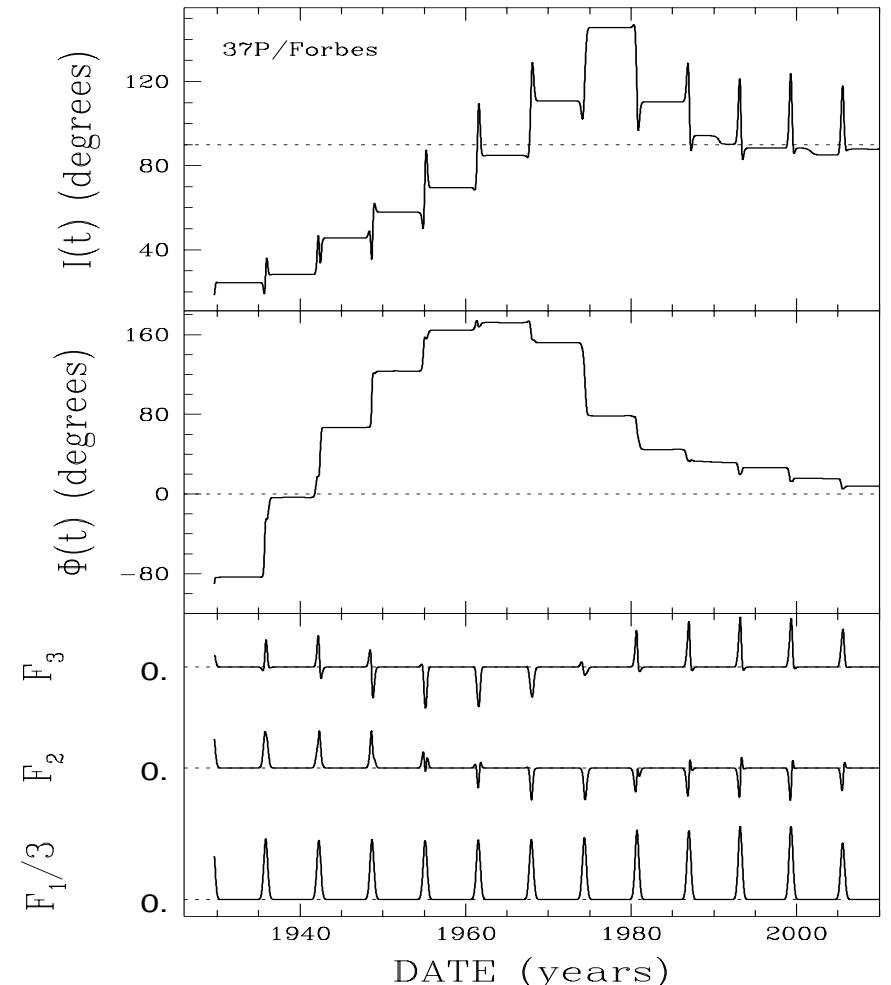

(e)

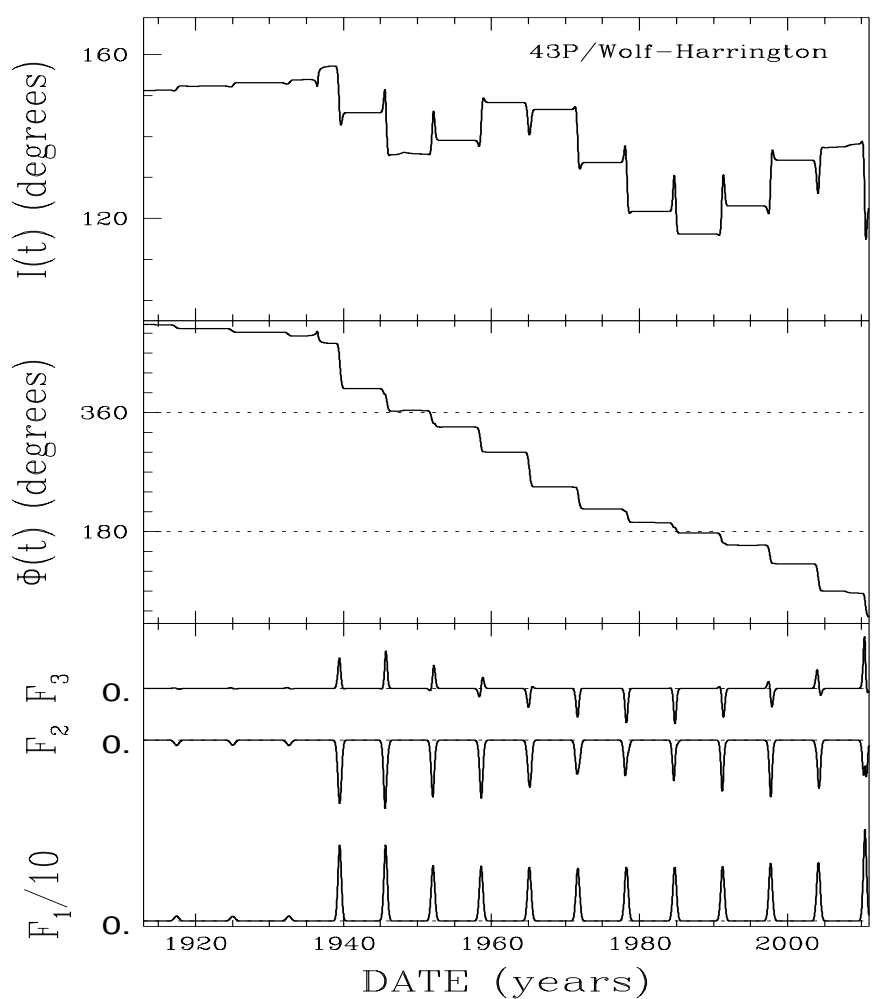

(f)

Fig. 4. continued

of $f_{\mathrm{p}}$ and oblateness $s$ for comets $31 \mathrm{P} /$ SchwassmannWachmann 2 and 21P/Giacobini-Zinner (Table 4b) represent the cometary model with the oblate spheroidal nucleus; the negative values of $f_{\mathrm{p}}$ and $s$ obtained for comets $43 \mathrm{P} /$ Wolf-Harrington, 37P/Forbes 16P/Brooks 2 and $32 \mathrm{P} /$ Comas Solá (Table $4 \mathrm{a}$ ) represent the prolate spheroid (the nucleus rotates around its longer axis). According to our solution the nucleus of $21 \mathrm{P} /$ GiacobiniZinner with oblateness along the spin-axis of about 0.29 (equatorial to polar radius of 1.41 ) is the most oblate among all six investigated comets and nucleus of $32 \mathrm{P} /$ Comas Solá with the ratio of equatorial to polar radius of 0.78 being the most prolate one.

The motion of the nucleus rotation axis represented by angles $I$ and $\phi$ and the qualitative variations of the nongravitational force components $F_{1}, F_{2}, F_{3}$ acting on the comet during its successive returns to the Sun are presented in Figs. 4a-f. According to our models six comets precess in different manner and at different rates; among them the precession of $21 \mathrm{P} /$ Giacobini-Zinner is the most rapid one. One can see that changes of $F_{1}, F_{2}$ and $F_{3}$ for all comets studied are significantly different. For comet Wolf-Harrington we notice a spectacular increase of nongravitational force after reducing the perihelion distance from 2.42 AU to 1.45 AU due to a close approach to Jupiter in 1936. In the Schwassmann-Wachmann 2 case two large changes of nongravitational force are visible; both due to a close encounter with Jupiter (March 1926 within $0.179 \mathrm{AU}$ and March 1997 within $0.246 \mathrm{AU}$ ): the first - before comet discovery, the second - after the last detection of the comet.

The present precession models impose some constraints on physical properties of the nuclei of the comets. Using solutions shown in Tables $4 \mathrm{a}-\mathrm{b}$ it is possible to calculate values of Sekanina's (1984) torque factor $f_{\text {tor }}=$ $f_{\mathrm{p}} / s$. Then we are able to calculate the value of $P_{\mathrm{rot}} / R_{\mathrm{a}}$ ratio from the formula: $P_{\text {rot }} / R_{\mathrm{a}}=4 \pi \cdot f_{\mathrm{p}} /(5 s)$, where the equatorial radius, $R_{\mathrm{a}}$, is related to the oblateness and the polar radius, $R_{\mathrm{b}}: R_{\mathrm{b}} / R_{\mathrm{a}}=1-s$. Our models give:

$$
P_{\text {rot }} / R_{\mathrm{a}}=\left\{\begin{aligned}
2.42 \pm 0.23 & \text { Comas Solá } \\
4.77 \pm 0.08 & \text { Giacobini-Zinner } \\
5.77 \pm 0.53 & \text { Brooks } 2 \\
12.51 \pm 2.36 & \text { Schwassmann-Wachmann } 2 \\
27.1 \pm 3.1 & \text { Wolf-Harrington } \\
31.6 \pm 1.0 & \text { Forbes }
\end{aligned}\right.
$$

where $P_{\text {rot }}$ is in hours and $R_{\mathrm{a}}$ in kilometers.

These relationships lie within the region occupied by comets with well-determined sizes and rotation periods (see Fig. 5). In Fig. 5 are also shown relationships between effective radii ${ }^{2}$ and rotation periods resulting from other investigations undertaken by Sitarski and collaborators for some short-period comets.

\footnotetext{
${ }^{2}$ Effective radius correspond to a sphere of the same projected area, which changes from $\pi R_{\mathrm{a}}^{2}$ to $\pi R_{\mathrm{a}} \cdot R_{\mathrm{b}}=\pi(1-s) \cdot R_{\mathrm{a}}^{2}$ according to the spin-axis orientation. Thus, we approximate the effective radius by a mean value of circles radii whose areas correspond to maximum and minimum projected areas of ellipsoidal nucleus, respectively.
} 


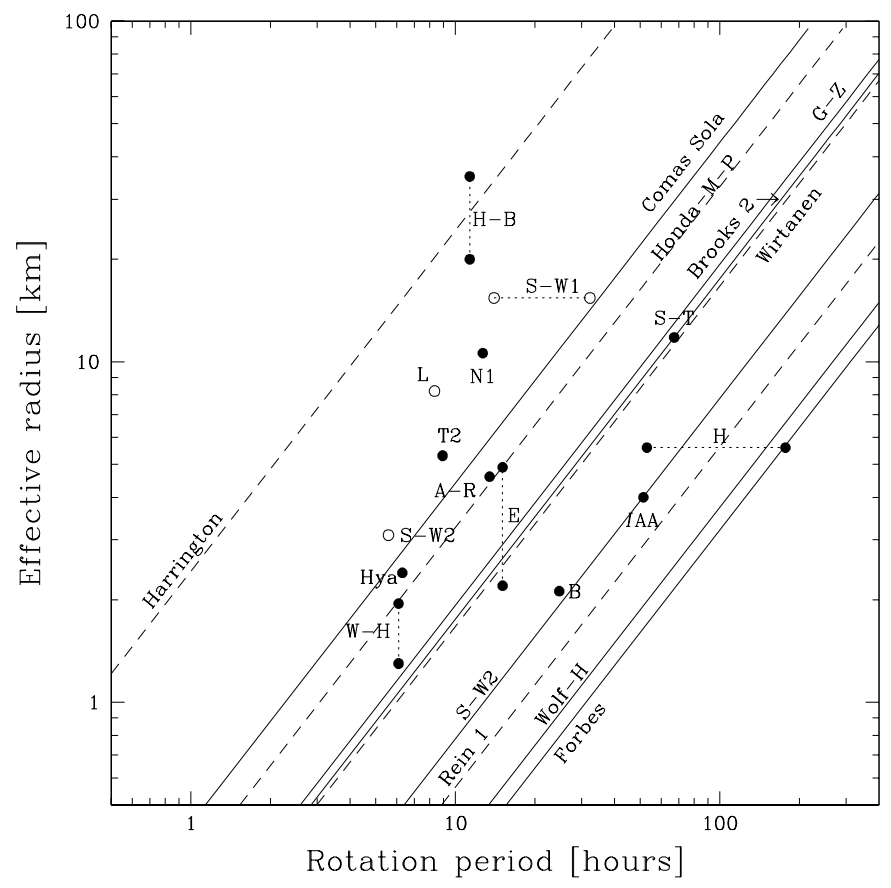

Fig. 5. Logarithm of the nucleus radius (e.g. effective radius) is plotted versus the logarithm of the rotational period for a sample of well-observed cometary nuclei. The plotted nuclei are $\mathrm{H}$ - Halley, S-T - Swift-Tuttle, W-H - WilsonHarrington, E - Encke, A-R - Arend-Rigaux, T2 - Tempel 2, N1 - Neujmin 1, S-W1 - Schwassmann-Wachmann 1, SW2 - Schwassmann-Wachmann 2, L - Levy, IAA - IRASAraki-Alcock, B - Borrelly, H-B - Hale-Bopp (C/1995 O1), Hya - Hyakutake (C/1996 B2); open circles denote the upper limits of the nuclear radius. Linear relationships for presented erratic comets are plotted by solid straight lines (where G-Z - Giacobini-Zinner). Analogous ralationships obtained for comets 30P/Reinmuth 1, 45P/Honda-Mrkos-Pajdušáková, 46P/Wirtanen and 51P/Harrington are shown by dashed lines

\section{Comparing the individual models with other studies}

\subsection{Periodic comet Comas Solá}

Comet 32P/Comas Solá was discovered in November 1926 and was observed extensively (from several months up to almost two years) during its all nine returns; last apparition was in 1995-97; next perihelion passage will occur in April 2005. No major orbital changes occurred during this time interval: an orbital period ranged between 8.5 and $9 \mathrm{yr}$ and perihelion distance somewhat varied near $1.8 \mathrm{AU}$ (see Table 2). This comet is classified as an erratic comet (Marsden et al. 1973; Sekanina 1993a) although the discontinuities in nongravitational perturbations of its orbital motions are less spectacular than for the Giacobini-Zinner. All orbital investigations of 32P/Comas Solá (Marsden et al. 1973; Forti 1983, 1989; KSS 1998b) have shown that the comet's deceleration reached maximum about 1940 and next fastly changed into acceleration. In other words, the tendency of $A_{2}$ to increase switched over to the tendency to decrease. This qualitative picture of the behaviour of $A_{2}$ may suggests that before the 1944 apparition something happened with cometary nucleus. Possibly an episodic outgassing event took place (Sekanina 1993a). This was confirmed by our numerical modelling of the real orbital motion of Comas Solá during 1926-1996. The best solution was obtained by setting the discontinuity of the time shift $\tau$ to appear at the moment of aphelion passage exactly in 1940 (KSS 1998b). This moment also coincides with the moment of transition from deceleration to acceleration visible in estimates of $A_{2}$ (see open circles in Fig. 3). In the previous paper on Comas Solá (KSS 1998b) we obtained two equivalent models representing oblate and prolate spheroidal nucleus. However, taking into account last astrometric observations carried out after perihelion passage in March 1996 (not included in KSS) it appears that corrected model (Table 3 and Fig. 4c) with prolate nucleus is better fitted to observations. Assumed discontinuity in $\tau$ seems to cancel the peak of Marsden's $A_{2}$ (full circles in Fig. 3). The mean $A_{2}$ was calculated here by averaging the values of $A_{2}$ weighted by a function $g\left(r^{\prime}\right)$ :

$\bar{A}_{2}=\int A_{2}(t) g\left(r^{\prime}\right) \mathrm{d} t / \int g\left(r^{\prime}\right) \mathrm{d} t$,

where the integrations are performed between given moments of two aphelion passages. These values of $\bar{A}_{2}$ are given as full circles and thick dashed horizontal lines in Fig. 3.

From CCD photometry taken at the heliocentric distance of 3.1 AU, Lowry et al. (1999) obtained an upper limit (because of coma contamination) of the nuclear radius of $3.2 \pm 0.4 \mathrm{~km}$. Thus, from our prolate forced precession model we obtain an upper limit for rotational period of $7.3 \mathrm{hr}$.

\subsection{Periodic comet Forbes}

Comet 37P/Forbes was discovered in August 1929 and is observed every 6.3 years except for its three returns in: 1935, 1955, and 1967. In Fig. 1, presenting the plots of comet's distances from the Sun and the Earth, one can see that there were unfavourable observational conditions during those three lost returns. In August 1990 the comet approached Jupiter to within 0.34 AU what caused some changes in orbital elements, especially in the angular elements (e.g. the inclination of the orbit plane increased from 4.7 to 7.2 .

There was no problem linking satisfactorily all observations of the comet to one system of orbital elements and seven nongravitational parameters, although Comet Forbes may be treated as an erratic comet since the $A_{2}$ value changed its sign after 1960 . This change of the secular deceleration into acceleration can be explained by the spin axis precession of the comet's nucleus as it is visible in Fig. 4. The nucleus of the comet appeared to be slightly prolate along the rotation axis, and the ratio of the polar radius to the equatorial one amounts to 1.06 . 


\subsection{Periodic comet Brooks 2}

The comet was discovered by Brooks in July 1889. It has been observed every seven years during consecutive returns to the Sun except for 1918 and 1967 when it was not rediscovered because of poor observational conditions (this is well documented in Fig. 1 where we plotted distances of the comet from the Sun and the Earth).

It was observed in 1889 that Comet Brooks 2 split into at least nine fragments. Then the principal comet was rediscovered in 1896 and has been observed during next 12 apparitions, the last time in 1994/95. The splitting was probably caused by an extremely close approach of the comet to Jupiter in 1886 to within 0.001 AU. It was carefully examined by Sekanina \& Yeomans (1985) who also investigated the nongravitational motion of the comet. It seems that Comet Brooks 2 is the continuously splitting object (though now not in such a spectacular manner as in 1889) and therefore nongravitational forces acting on the comet are among the largest for short-period comets.

Values of the parameter $A_{2}$, as determined from observations of three consecutive apparitions, show irregular variations in time. Sekanina \& Yeomans (1985), analysing also the light curves of the comet, tried to fit such parameters of the rotating comet's nucleus to predict the observed variations of $A_{2}$. They found a nearly spherical, slowly precessing, nucleus (they assumed the oblateness of $0.01)$.

We obtained our solution for the forced precessing nucleus by linking all the apparitions of Comet Brooks 2 . The varying activity of the comet was simulated by four different values of $\tau$ for different intervals describing an asymmetry of the function $g(r)$ with respect to perihelion. Values of all the remaining parameters have been obtained by the least squares solution of 961 observational equations. In our model the nucleus of the comet appeared to be slightly prolate along the spin axis, the ratio of the polar radius to the equatorial one amounts to 1.06 . It is interesting that our determined value of the lag angle $\eta=21.35$ is very close to that assumed by Sekanina \& Yeomans (1985); also their value of the torque factor is comparable with ours: $f_{\text {tor }}=f_{\mathrm{p}} / s=0.1410^{8}$. However, mean values of $A_{2}$ calculated for every perihelion time from our model of the nongravitational motion have rather regular run with time and do not contain such jumps as in case of $A_{2}$ determined by linking of three consecutive apparitions, presented in Table 3 and in Fig. 3. We also should notice that the plot of the nucleus radius versus rotational period for Comet Brooks 2 lies very well among the plots for other periodic comets in Fig. 5. Radius of the comet's nucleus was estimated by Sekanina \& Yeomans (1985) as $0.4 \mathrm{~km}$, which gives the rotation period of 2.3 hours, what seems to be unacceptably short. On the other hand, if we take following Sekanina \& Yeomans (1985), the rotation period equal to 5.69 hours, we obtain the equatorial radius of the comet's nucleus about $1 \mathrm{~km}$.

\subsection{Periodic comet Wolf-Harrington}

The comet 43P/Wolf-Harrington was discovered twice. First time it was observed by M. Wolf in December 1924 and only after twenty seven years in October 1951 it was rediscovered by R. G. Harrington. The identity of both comets was supported by Wiśniewski (1964). Between 1924 and 1951 the comet three times returned to the Sun and approached Jupiter twice: in June 1936 to within $0.123 \mathrm{AU}$ and in January 1948 to within 0.716 AU. These approaches caused considerable changes in the comet's orbit. After 1936 the perihelion distance was reduced from 2.43 to $1.45 \mathrm{AU}$ and the orbital period dropped from 7.6 to 6.2 years. The second encounter to Jupiter caused the opposite changes but much smaller then the first one. Since 1952 the comet has been observed at every return to perihelion every 6.5 years. The perihelion distance of the comet will decrease into $1.36 \mathrm{AU}$ and its orbital period will reach 6.1 years in the 2010 apparition due to approach Jupiter in June 2007. The orbit evolution is shown in Fig. 1.

The orbital motion of the comet is subjected to variable nongravitational perturbations (Szutowicz 1987, 1992). The Marsden's nongravitational acceleration was slightly decreasing until 1978 and then rapidly changed reaching value close to zero (see Table 3 and open circles in Fig. 3). The general tendency of this variation was no longer kept and the acceleration started to increase after the next apparition in 1984.

Preliminary linkage of all comet's apparitions by the forced precession model proved that the model should be enriched by additional parameters (Królikowska et al. 1998a, 1998b). The forced precession model was reasonably fitted to all observations when two changes of the time shift of the function $g\left(r^{\prime}\right)$ with respect to the perihelion were assumed. Thus, our orbital solution is represented by six basic precessional parameters and three values of time shifts (see Table 4). It is interesting that evolution of the perihelion shift of $g\left(r^{\prime}\right)$ from negative to positive and again negative number of days, has been confirmed by a model of other type. All observations of the comet have been also successfully linked by the spotty nucleus model where discontinuities in the nongravitational perturbations are connected with a surface redistribution of the active areas (Szutowicz 2000). The moments of discontinuities in $\tau$ roughly coincide with time of activation and deactivation of discrete sources of a gas emission established on the basis of the spotty nucleus model. It seems that the irregular perihelion shifts of the maximum activity play an essential role in the nongravitational perturbations of this comet. It should be noticed that the asymmetric model of outgassing for the precessing nucleus of the comet Wolf-Harrington essentially changed the meaning of transverse component of the nongravitational force. As it follows from Fig. 3 the parameter $A_{2}$ is not so strongly variable with time as in the case of symmetric model. 
There are not too many nuclear radius estimates of the comet 43P/Wolf-Harrington. Lowry et al. (1999) derived the nuclear radius of $3.3 \mathrm{~km}$ from photometric observations of the comet at a heliocentric distance of $4.87 \mathrm{AU}$. They also estimated the lower limit of the nuclear radius of $0.42 \mathrm{~km}$ based on the measured $\mathrm{OH}$ production rates given by A'Hearn et al. (1995). Based on this last value our forced precession model gives a lower limit of rotational period of $11.4 \mathrm{hrs}$.

\subsection{Periodic comet Schwassmann-Wachmann 2}

This comet is a particularly appropriate candidate for a detailed investigation of nongravitational forces because of two observational facts: 1.) it has been observed at all eleven apparitions since its discovery in 1929; 2.) the astrometric material covered the substantial arcs of revolution periods at almost each return (in the last three apparitions even more than $2.5 \mathrm{yr}$ out of the orbital period of $6.5 \mathrm{yr}$ ). This comet was discovered after its close encounter with Jupiter (within 0.179 AU) when the perihelion distance reduced from 3.56 AU to 2.09 AU, the eccentricity increased from 0.19 to 0.39 and orbital period shortened from 9.3 yrs to 6.4 yrs (see Table 2). In March 1997 - after the last comet detection - the second close approach to Jupiter occurred (within 0.246 AU) which moved comet back to the larger perihelion distance of $3.41 \mathrm{AU}$; next perihelion passage will occur in 2002. During almost 70 years of observations only moderate orbital changes were detected: the perihelion distance varied between 2.09 AU (1929) - 2.16 AU (1961) - 2.07 AU (1994). Nevertheless, this comet, in spite of rather large perihelion distance, exhibits strong variations of $A_{2}$ and additionally belongs to a group of comets with largest negative (and absolute) values of $A_{2}$ (see Fig. 2 in Sekanina 1993a).

Sekanina (1993b) investigated this comet assuming outgassing from a few isolated active areas. His analysis was based on observed light curves and eight discrete values of nongravitational parameter $A_{2}$ derived from orbital calculations. The apparent light curves of consecutive apparitions were compiled in four groups: 1929-35, 1942-48, 1955-68, 1974-87. He found a possible solution with four active sources: one persistent and three shortlived appearing in 1929, 1955 and 1961. Forced precession model presented in Table 4 requires two discontinuities in time shift of maximum of activity, however occurring not before 1968 perihelion passage. Thus, seven former appearances carried out between 1929-68 could be linked to a single set of seven parameters $A, \eta, I_{0}, \phi_{0}, f_{\mathrm{p}}, s$ and $\tau$. Before 1970 and after 1978 aphelion passages we derived small positive time shifts about 3.3 days and 1.2 days, respectively, in significant contrast to a large time shift of about 62 days during 1974 perihelion passage (e.g. in time interval between 1970-1978). These discontinuities can be interpreted as some sudden changes in the geometry of activity related to the activation of a new discrete source and disappearance/decline of some old one; however global cometary activity (described by parameter $A$ in forced precession model) can remain unaltered.

Unfortunately, it is not possible to verify such large temporal changes with observed light curves because only on five apparitions: 1929, 1942, 1955, 1968 and 1981 (Fig. 4 in Sekanina 1993b) the comet was observed photometrically for several months around the perihelion. In the remaining returns the comet was detected for many months before and many months after perihelion passages. Taking into account more complete light curves around perihelion passage at 1929, 1942, 1955 it seems that maximum occurred slightly before these passages. However, we do not have any information about the position of maximum light curves in 1935, 1948 and 1961 perihelion passages, which contributed to our slightly positive value of time shift before 1970 .

Orbital calculations based on Marsden's $A_{1}, A_{2}, A_{3}$ show local maximum of $A_{2}$ around 1974 perihelion passage. This coincides with two model discontinuities of $\tau$ at 1970 and 1978 aphelion passages which are essential in linking all eleven returns of $31 \mathrm{P} /$ SchwassmannWachmann 2 (hereafter SW2). As in the Comas Solá case, assumed discontinuities of $\tau$ cancel this local peak of Marsden's $A_{2}$ (compare open circles with full ones in Fig. 3).

From the near aphelion CCD photometry of SW2 Luu \& Jewitt (1992) found a best-fit lightcurve period of $5.58 \pm 0.03 \mathrm{hr}$ which they interpreted as the rotation period of the nucleus. Since the geometric albedo of the nucleus of SW2 is unknown, Luu \& Jewitt assumed a typical albedo for other known nuclei of 0.04 , which corresponds to an upper limit (because of the presence of coma in their data) of effective radius of $3.1 \mathrm{~km}$. Next, from about 0.5 magnitude variation of the lightcurve they conclude that the axis ratio $R_{\mathrm{a}} / R_{\mathrm{b}}$ (equatorial to polar radius) must be greater than 1.6 (rather extreme asphericity). For such nucleus parameters Luu \& Jewitt derived the critical density (resulting from assumption that nucleus must be stable against centripetal disruption) of about $460 \mathrm{~kg} \mathrm{~m}^{-3}$. They conclude that this value is greater than other cometary estimates of mean density (see Fig. 6 therein). In fact substituting their values of $P_{\text {rot }}$ and axis ratio into Eq. (9) in their paper we obtain even greater value of $530 \mathrm{~kg} \mathrm{~m}^{-3}$. (It seems that $460 \mathrm{~kg} \mathrm{~m}^{-3}$ corresponds to axis ratio of about 1.4.) Our model is in some contradiction with axis ratio postulated by Luu \& Jewitt. Our best forced precession model gives slightly oblate nucleus with a ratio $R_{\mathrm{a}} / R_{\mathrm{b}}$ of $1.10 \pm 0.03$ what leads to a smaller critical density of about $380 \mathrm{~kg} \mathrm{~m}^{-3}$ for the rotation ratio of $5.58 \mathrm{hr}$. This still rather high density is mainly due to a short rotation ratio. Substituting this value of rotation period into $P_{\text {rot }} / R_{\text {a }}$ relation, our model of SW2 requires very small effective radius of $0.40 \mathrm{~km}$ (equatorial radius of $0.41 \mathrm{~km}$ ). However, A'Hearn et al. (1995) from two near-aphelion observations of SW2 in 1981 estimated a water production rate of $\log Q\left[\mathrm{H}_{2} \mathrm{O}\right]=27.85$, which corresponds to an active area of $7.9 \mathrm{~km}^{2}$. This gives a lower limit on the nucleus radius of $0.8 \mathrm{~km}$ with the 
assumption that a whole surface is active. Since SW2 recently undergone large reduction of perihelion distance thus it should be significantly more active than others short-period comets. Therefore, divergence from the forced precession model based on rich positional material could be interpreted as resulting from underestimation of coma contamination in photometric data of SW2.

\subsection{Periodic comet Giacobini-Zinner}

The comet was discovered in December 1900 by Giacobini, and rediscovered in October 1913 by Zinner. Its return to the perihelion in 1907 was lost for observers because of poor observational conditions, what is very well visible in Fig. 1. Because of the unfavourable position of the comet with respect to the Earth and Sun, the comet was not observed in 1920 and 1953 too; in October 1939 only two observations have been made, and five observations in September 1965 (made at one observatory) did not fit, unfortunately, to any of our complete numerical models of the comet's motion and have had to be rejected. It is interesting that Comet Giacobini-Zinner could be a candidate for the Earth-comet collision since the ascending node of the comet's orbit lies almost in the Earth's orbit, and in December 1926 the minimum distance between orbits of both celestial bodies amounted to 0.0009 AU only. In 1985 the International Cometary Explorer reached the comet beyond $7800 \mathrm{~km}$ from the nucleus. However, during the comet fly-by only physical observations of cometary tails were made.

Nongravitational effects in the motion of Comet Giacobini-Zinner show an irregular behaviour in time if we observe values of the parameter $A_{2}$ as determined by linking of three consecutive apparitions of the comet (see Table 3): in the period 1900-1999 $A_{2}$ changed its sign after 1960. It was also shown that the time-shift $\tau$ plays an essential rôle when investigating the nongravitational motion of the comet (Yeomans \& Chodas 1989; Sitarski 1994a), and that $\tau$ changed its sign after 1960 too.

Sekanina (1985) carefully examined the nongravitational motion of Comet Giacobini-Zinner trying to explain its "erratic" character by the precessional motion of spin axis of the comet's nucleus. However, Sekanina has had to assume the unrealistically large oblateness of nucleus equal to 0.88 . In our forced precession model for the comet's motion we obtained the reasonable oblateness $s=0.29$, and got a small lag angle $\eta=6.5$ in some agreement with Sekanina who assumed $0^{\circ}$ for the $\eta$ value. An irregular nongravitational behaviour of the comet results in the complicated numerical model of the comet's motion: we obtained two values of the nongravitational parameter $A$ : $A^{(1)}$ before and $A^{(2)}$ after 1956, and four different values of $\tau$ with different signs (we got the positive values for $\tau_{1}$ and $\tau_{2}$ and negative ones for $\tau_{3}$ and $\tau_{4}$ ). This model successfully links all observations of 1900-1999. We notice that the mean values of $A_{2}$ calculated from our model show quite regular run with time as it is seen in
Fig. 3. On the other hand the wobbling of the nucleus spin axis is more rapid than that in Sekanina's results (see $I(t)$ in Fig. 4a), and variations of $\phi(t)$ and $F_{3}$ express an extremely speedy spin axis precession. The ratio of the nucleus radius to the rotational period for Comet Giacobini-Zinner, resulting from our solution, has a very good position in Fig. 5 .

Assuming rotational period of about $9.5 \pm 0.2 \mathrm{hr}$ detected by Leibowitz \& Brosch (1986), our forced precession model gives equatorial radius of $1.99 \pm 0.08 \mathrm{~km}$ and polar radius of $1.41 \pm 0.07 \mathrm{~km}$. Belton (1991), however, suggests that periodicity found by Leibowitz \& Brosch (1986) refers to second harmonic giving the probable period of about $19 \mathrm{hr}$. This period provides twice as large nucleus with effective radius of $3.7 \mathrm{~km}(4.0 \mathrm{~km}$ and $2.8 \mathrm{~km}$ for equatorial and polar radius, respectively).

\section{Conclusions and final remarks}

The main conclusions can be summarized as follow:

1. The forced precession model of the rotating cometary nucleus successfully describes a long-term motion of short-period comets, even "erratic" comets strongly affected by nongravitational forces. In five of six erratic comets, however, we have detected certain changes in the geometry of the sublimation area on the nucleus surface. In our formalism we modelled such changes by varying the time shift parameter, $\tau$, which represents the moment of maximum activity with respect to perihelion time (see Table 4). Additionally, in the $21 \mathrm{P} /$ Giacobini-Zinner case we must assume that the global activity of comet (represented by a parameter $A$ in our model) has changed between two consecutive perihelion passages in the years 1946-1959. It was the only way to link all the observed apparitions to a single consistent model. All these necessary assumptions involved additional model parameters which should be determined from positional data. Thus, the forced precession models for "erratic" comets, except $37 \mathrm{P} /$ Forbes case, are more complicated than for "normal" comets.

2. The asymmetric model of outgassing with variable time shifts (e.g. solutions with more than one $\tau$ ) caused an essential modification of temporary $A_{2}$ values. The best examples are here $21 \mathrm{P} /$ Giacobini-Zinner and 43P/Wolf-Harrington. It is clearly seen in Fig. 3 where we should compare the open and black dots. In such cases, however, the interpretation of the parameter $A_{2}$ is more complicated. Thus, values of nongravitational parameters determined as "constants" by linking three consecutive apparitions should be treated as very uncertain. In general, forced precession models reveal that temporal variations of $A_{2}$ are considerably tempered for investigated "erratic" comets.

3. Forced precession model gives some important information about shape and other physical properties of the cometary nucleus. These are oblateness of the nucleus and value of $P_{\text {rot }} / R_{\mathrm{a}}$ ratio or $P_{\text {rot }} / R_{\mathrm{e}}$ ratio, where $R_{\mathrm{e}}$ is the equivalent radius. 
Among investigated comets are comets with equatorial radius, $R_{\mathrm{a}}$, greater than polar radius, $R_{\mathrm{b}}$, (models with positive parameter $s$ in Table 4 meaning oblate spheroidal shape) as well as comets with the equatorial radius smaller than the polar one (prolate spheroidal shape of nucleus is represented by negative $s$ ). This is in a full agreement with observations which indicate the existence of elongated comets (for example 49P/Arend-Rigaux with probable axial ratio of $R_{\mathrm{b}} / R_{\mathrm{a}}=1.59$ and $19 \mathrm{P} /$ Borrelly with $\left.R_{\mathrm{b}} / R_{\mathrm{a}}=2.55\right)$.

The range of $P_{\text {rot }} / R_{\mathrm{a}}$ ratios derived from forced precession models is also in a very good agreement with observations. If we draw lines representing these ratios on the rotational period vs. radius diagram, it turned out that all of them lie among the plotted comets with well-known sizes and rotational periods (see Fig. 5).

Acknowledgements. The authors would like to thank Professor Andrzej Wernik for his contributions to improve this paper. This work was supported by the Polish Committee for Scientific Research (the KBN grant 2.P03D.002.09).

\section{References}

A'Hearn, M. F., Millis, R. L., Schleicher, D. G., Osip, D. J., \& Birch, P. V. 1995, Icarus, 118, 223

Belton, M. J. S. 1991, ed. R. L. Newburn, M. Neugebauer, \& J. Rahe, in Comets in the Post-Halley Era

Bielicki, M., \& Sitarski, G. 1991, Acta Astron., 41, 309

Forti, G. 1983, A\&A, 126, 307

Forti, G. 1989, A\&A, 215, 381

Królikowska, M., \& Sitarski, G. 1996, A\&A, 310, 992

Królikowska, M., Sitarski, G., \& Szutowicz, S. 1998a, Acta Astron., 48, 91
Królikowska, M., Sitarski, G., \& Szutowicz, S. 1998b, A\&A, 335,757

Królikowska, M., Sitarski, G., \& Szutowicz, S. 1999, in Evolution and Source Regions of asteroids and Comets, ed. J. Svoren, E. M. Pittich, \& H. Rickman, Proc. IAU Coll., 173,381

Królikowska, M., \& Szutowicz, S. 1999, A\&A, 343, 997

Leibowitz, E. M., \& Brosch, N. 1986, Icarus, 68, 430

Lowry, S. C., Fitzsimmons, A., Cartwright, I. M., \& Williams I. P. 1999, A\&A, 349, 649

Luu, J. X., \& Jewitt, D. C. 1992, AJ, 104, 2243

Marsden, B. G., \& Sekanina, Z. 1971, AJ, 76, 1135

Marsden, B. G., \& Williams, G. V. 1997, Catalogue of Cometary Orbits, Twelfth ed.

Marsden, B. G., Sekanina, Z., \& Yeomans, D. K. 1973, AJ, 83,64

Rickman, H., \& Jorda, L. 1998, Adv. Space Res., 21, 1491

Sekanina, Z. 1984, AJ, 89, 1573

Sekanina, Z. 1985, AJ, 90, 827

Sekanina, Z., Yeomans, D. K. 1985, AJ, 90, 2335

Sekanina, Z. 1988, AJ, 96, 1455

Sekanina, Z. 1993a, AJ, 105, 702

Sekanina, Z. 1993b, A\&A, 271, 630

Sitarski, G. 1979, Acta Astron., 29, 401

Sitarski, G. 1984, Acta Astron., 34, 53

Sitarski, G. 1992, Acta Astron., 42, 59

Sitarski, G. 1994a, Acta Astron., 44, 91

Sitarski, G. 1994b, Acta Astron., 44, 417

Szutowicz, S. 1987, Acta Astron., 37, 179

Szutowicz, S. 1992, in Proc. of the 30th Liege Int. Astroph. Colloquium, 371

Szutowicz, S. 1999, Ph.D. Thesis, in polish

Szutowicz, S. 2000, A\&A, 363, 323

Wiśniewski, W. 1964, Acta Astron., 14, 130

Yeomans, D. K., \& Chodas, P. W. 1989, AJ, 98, 1083 